\section{The Duncan memorial lecture}

Dr Duncan was the first medical officer of health in Britain and is remembered in many ways including an annual lecture in the city which benefited from his vision and experience, Liverpool. We are pleased to be able to publish the 1993 lecture which is the second in our lecture series following the 1993 Cochrane lecture published earlier this year.

\section{Social inequalities and accidents}

Several articles pick up the theme of social inequalities and health and the three short reports focus on accidents of various types in the old and the young. Short reports continue to be a way of communicating results much more quickly than normal, especially since the number of papers submitted to the Journal each year has dramatically increased lately.

\section{Health expectancy compared with life expectancy}

A methodological article presents the case for adding health to years in calculations of expected life span - a rather different approach to quality of life - but advises caution in using and interpreting various methods of calculation.

\section{The UK Society for Social Medicine}

We have a long-established tradition of publishing abstracts of lectures and posters accepted by peer review for the Annual Scientific Meeting of this Society which numbered in its members several of the founders of this Journal. Although this is a special connection we welcome the submission of abstracts from other similar societies.

\title{
Editorial
}

\section{Why we need qualitative research}

"The almost sole recognition given to quantitative methods has trained students inadequately, established flawed standards of practice and research, and delayed the development of essential medical knowledge.... When qualitative methods are clearly established in our research repertoire, the advance of medical knowledge will be greatly accelerated". 1

Aetiological and health services research are dominated by quantitative methods: research tends to be considered real and serious only when it uses these approaches. Quantification has acquired a bogus value - if something can be measured or counted it gains a scientific credibility often not afforded to the unmeasured or unmeasurable. Because of this, a finding or result is more likely to be accepted as a fact if it has been quantified than if it has not. On occasions, our love affair with numbers goes even further: sometimes we may suspend our critical faculties when faced with quantitative information, whether derived from routine or ad hoc sources. As a result, many well known, widely accepted "facts" of doubtful accuracy have become entrenched in our supposed knowledge of health, disease, and health care, such as that one couple in 10 is infertile, one man in 10 is homosexual, one hospital bed in 10 in the USA is for intensive care, and the prevalence of coronary heart disease rose and is now declining. The scientific bases for all these claims are doubtful.

We clearly need and can benefit enormously from the quantification of many aspects of the physical, social, and psychological worlds. Indeed, it is the undeniable importance of quantitative enquiry that makes the need for improvements in its conduct so crucial. This can be achieved in three ways. Firstly, by the development of more sophisticated statistical methods for handling quantitative data. Secondly, by using quantitative methods in combination with qualitative methods. And thirdly, by acknowledging that some situations are inevitably beyond the scope of quantitative methods but could be investigated more appropriately by qualitative ones.

\section{What is qualitative research?}

Before considering how qualitative research might help, what exactly is it? As the opening quotation suggests, many scientifically trained researchers are unaware of qualitative methods and some even take pride in their ignorance. It is easiest to start with some widely held misconceptions about qualitative research: it is not about the measurement of quality; done properly, it is no less rigorous or objective than quantitative work; it is not an easy haven for innumerate scientists; and it is not simply research using samples that would be too small for statistical analyses.

In essence it is research that helps us to understand the nature, strengths, and interactions of variables. Like quantitative research, it can address causation and it involves observation and interpretation of events. Unlike quantitative research, it seeks to answer the "what" question, not the "how often" one. Thus, rather than adopting a simplified, reductionist view of the subject in order to measure and count the occurrence of states or events, qualitative methods take an holistic perspective which preserves the complexities of human behaviour. ${ }^{2}$ A qualitative approach interviews, observation of activities, interpretation of written material - is most revealing when the variables of greatest concern are unclear. The focus may range from a single individual or small group, such as the interaction between a health professional and a patient, to the functioning of a large organisation, such as a hospital. The benefits of qualitative methods are greatest when the subject of study cannot be controlled and is poorly defined. They have the capacity to reveal what is going on but do not attempt to measure how often an event or association occurs. Given these attributes, how can qualitative research contribute to our understanding of health and health care? 
Qualitative combined with quantitative research

Qualitative methods may enhance quantitative research in four ways. Firstly, quantitative results are often misleading because insufficient attention is paid to the way the data were created such as how factors were defined, the extent of adherence to those definitions, and how the data were collected. Used in combination, qualitative methods can help to improve the accuracy and relevance of quantitative studies by increasing our understanding of the creation of quantitative data, an activity known as ethnostatistics.

For example, it is reported that Greece spends only 5.6\% of its gross national product on health services yet enjoys relatively good measures of health when compared with other OECD countries. This apparent enigma can be partly explained by the failure of the OECD to take into account the black market in health care in Greece which has been estimated to contribute an additional $30 \%$ to the official expenditure (Dunning A - personal communication). Routine mortality data can also be misleading - the internationally reported infant mortality rate for France is an underestimate as it excludes neonatal deaths in babies weighing less than $500 \mathrm{~g}$ at birth as these are defined as stillbirths. And imprecision in defining the cause of death has rendered much observational quantitative research on coronary heart disease suspect. ${ }^{34}$ The current belief that mortality from coronary heart disease has declined by about $30 \%$ in several developed countries may simply reflect secular changes in diagnostic choices just as the earlier supposed rise in incidence may have been an artefact. Meanwhile, both supposed "facts" have influenced the direction of much research on the prevention of the disease.

A simple acceptance of the accuracy of quantitative data is not confined to the use of routine information. Several years ago an elegant study carried out in the East End of London demonstrated the differences between people's responses to questionnaires about their health and the information they confided in a researcher who visited them regularly and carried out largely unstructured informal interviews. ${ }^{5}$ Whereas the questionnaires revealed what the respondents perceived was the correct view to express to professionals, the interviews discovered their actual private experiences and opinions. Such survey results are therefore likely simply to reinforce existing views held by health professionals, researchers, and policy makers and hence confirm the status quo.

The second way qualitative research can assist quantitative work is by identifying the appropriate variables to be measured. Too often people start measuring before they fully understand the underlying issues and thus may not target the appropriate factors. For example, in the last few years there has been considerable interest in encouraging doctors to audit their work. To promote this, many quantitative surveys were carried out to find out the views of doctors. Needless to say, when confronted with closed questions which sought their views of audit, most doctors responded in the politically correct way and stated that they were in favour of peer review and other such noble ideals. Meanwhile, observational reports showed that little or no audit was taking place. This apparent contradiction between intent and action was explained by a qualitative enquiry involving detailed interviews with doctors of all grades and observation of clinical meetings. ${ }^{6}$ This revealed 19 reasons why doctors were uncertain or unsupportive of audit, most of which had not been addressed in the previous quantitative studies. It suggested that unless the underlying anxieties and concerns were addressed, the introduction and acceptance of audit would continue to be unsuccessful.

The other two ways a qualitative approach can combine with quantitative methods are in providing explanations for unexpected or unexplained findings in quantitative studies and as a fertile source of hypotheses for quantitative enquiry.

\section{Qualitative instead of quantitative research}

Qualitative research can also contribute to our understanding, not by assisting and improving quantitative work but by replacing it. There are some subjects that are better investigated using a qualitative approach. These tend to be complex situations where the relevant variables associated with an outcome are not apparent. This type of research aims to increase our understanding of what is going on. An example may serve to clarify.

Waiting lists for surgery are of great public and professional concern. Most research that has been conducted on waiting lists has been quantitative and has sought methods of reducing their size or the waiting time. Despite these studies, waiting lists in the UK have increased. The lack of impact of quantitative research may not have been due to poorly conducted studies but rather to the use of a false idea of the nature of waiting lists. Traditionally, lists have been viewed by patients and professionals as akin to a bus queue in which someone joins the end and works their way to the front. A qualitative study involving interviews with patients, doctors, and administrative staff and observation of hospital records departments led to the development of a different conceptual model which seemed to be a closer reflection of the reality of waiting lists and therefore had the potential to provide a basis for designing a successful intervention to improve the management of patients. ${ }^{6}$ No amount of quantitative research based on the traditional model is likely to have had much benefit.

\section{Conclusions}

More frequent use of qualitative methods will greatly enhance both aetiological and health services research. Failure to use them more has retarded the advance in medical knowledge and at times led to false trails being followed. This has partly arisen because of scepticism on the part of quantitative scientists about the objectivity and rigor of qualitative methods. While this view may be justified occasionally, it is not a valid reason for ignoring the potential use of qualitative methods. As the statistician $\mathrm{J} \mathrm{W}$ Tukey pointed out, "far better an approximate answer to the right question .... than an exact answer to the wrong question." pursuing the measurable rather than the relevant. This would have serious consequences for our understanding both of the causes of diseases and how to improve health services.

NICK BLACK Reader in Public Health Medicine, Health Services Research Unit, London School of Hygiene and Tropical Medicine Member of the FECH Editorial Committee

1 Holman HR. Qualitative inquiry in medical research. $f$ Clin Epidemiol 1993;46:29-36.

Strong PM. The case for qualitative research. International fournal of Pharmacy Practice 1992;1:185-6.

3 Stehbens WE. Imprecision of the clinical diagnosis of coronary heart disease in epidemiological studies and atherogenesis. F Clin Epidemiol 1991;44:9991006 .

4 Burnand B, Feinstein AR. The role of diagnostic inconsistency in changing rate 40.

5 Cornwell J Hard-earned lives. London: Tavistock Publications, 1984.

5 Cornwell J. Hard-earned lives. London: Tavistock Publications, 1984. Sci Med 1993;36:849-56.

7 Pope C. Trouble in store: some thoughts on the management of waiting lists. Sociology of Health and Illness 1991;13:193-212.

8 Tukey JW. The future of data analysis. Annals of Mathematics and Siatistics 1962;33:1-67. 